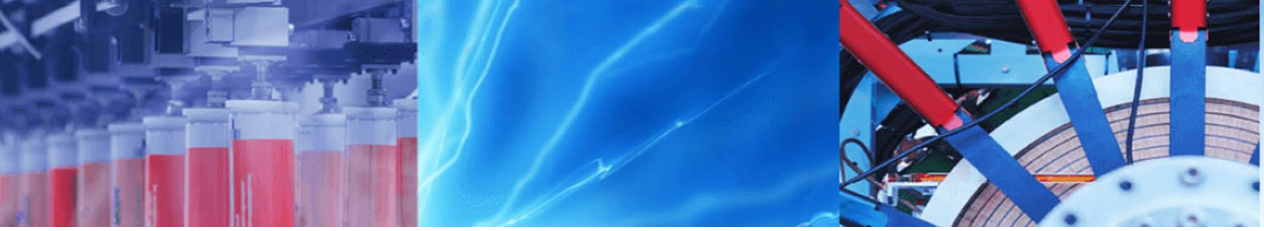

Research Article

\title{
Machine learning for energy consumption prediction and scheduling in smart buildings
}

\author{
Safae Bourhnane ${ }^{1}$ (D) Mohamed Riduan Abid ${ }^{2} \cdot$ Rachid Lghoul $^{2} \cdot$ Khalid Zine-Dine $^{3} \cdot$ Najib Elkamoun $^{1}$. \\ Driss Benhaddou ${ }^{4}$
}

Received: 14 October 2019 / Accepted: 9 January 2020 / Published online: 30 January 2020

(c) Springer Nature Switzerland AG 2020

\begin{abstract}
Predicting energy consumption in Smart Buildings (SB), and scheduling it, is crucial for deploying Energy-efficient Management Systems. Most important, this constitutes a key aspect in the promising Smart Grids technology, whereby loads need to be predicted and scheduled in real-time to cope for the strongly coupled variance between energy demand and cost. Several approaches and models have been adopted for energy consumption prediction and scheduling. In this paper, we investigated available models and opted for machine learning. Namely, we use Artificial Neural Networks (ANN) along with Genetic Algorithms. We deployed our models in a real-world SB testbed. We used CompactRIO for ANN implementation. The proposed models are trained and validated using real-world data collected from a PV installation along with SB electrical appliances. Though our model exhibited a modest prediction accuracy, which is due to the small size of the data set, we strongly recommend our model as a blue-print for researchers willing to deploy real-world SB testbeds and investigate machine learning as a promising venue for energy consumption prediction and scheduling.
\end{abstract}

Keywords Smart Grids · Smart buildings · Renewable energy · ANN · GA · CompactRIO

\section{Introduction}

Smart Grids (SG) have emerged as a solution to the increasing demand on energy worldwide. The grid refers to the traditional electrical grid that is a collection of transmission lines, substations, and other components that make sure energy is delivered from the power plant to the home or business [1]. The smartness in the SG resides in the twoway communication between the utility and the customers, in addition to the sensing along the lines. The main components of a SG are controls, computers, automations, in addition to other new technologies that are working together to accommodate for the quick increase in the energy demand. The SG has many benefits among which we state: more efficient energy transmission, improving security, reducing peak demand which helps with the decrease of electricity rates, etc. SG are also known by the use of renewable energy sources.

The prediction and scheduling are two of the main pillars of efficient Energy Management Systems (EMS). EMSs are very crucial for the well-functioning of the SG. They are responsible for managing the power flux within the SG elements in order to minimize the costs and optimize the quality [2].

The prediction of the energy consumed by different appliances is one of the building blocks of the concept of SGs. The energy consumption can be seen as a nonlinear time series with a number of complex factors [3]. With many renewable energy sources used in the SGs, the energy prediction methods are getting more and more

$\checkmark$ Safae Bourhnane, s.bourhnane@aui.ma; Mohamed Riduan Abid, r.abid@aui.ma; Rachid Lghoul, r.lghoul@aui.ma; Khalid Zine-Dine, khalid.zinedine@um5.ac.ma; Najib Elkamoun, elkamoun.n@ucd.ac.ma; Driss Benhaddou, dbenhaddou@uh.edu|'12AROSERI Lab., Faculty of Sciences, Chouaib Doukkali University, El Jadida, Morocco. ${ }^{2}$ School of Science and Engineering, Al Akhawayn University, Ifrane, Morocco. ${ }^{3}$ Mohammed V University, FSR, Rabat, Morocco. ${ }^{4}$ School of Engineering and Technology, University of Houston, Houston, USA. 
accurate, and hence, the prediction becomes a crucial part in the efficient planning of the entire SG.

There are different approaches that are used for the prediction of the energy consumption. The most popular ones use machine learning (ML).

Machine learning (ML) is one of the growing technical fields that merge between computer science and statistics. It tackles the issue of building computers that learn through experiences and hence provide more improved algorithms. ML keeps witnessing advances thanks to the new algorithms and the availability of online data, in addition to the accessibility of the computing power [4]. Artificial Neural Networks (ANN) are one of the ML algorithms are widely used in this context.

ANNs have seen light in the early 1940s but have not been widely used until lately. They became very popular thanks to the outstanding results they offer. They are very powerful with large datasets which gives the neural network enough data to train the model. In brief, ANNs are inspired by the way the brain processes information. They build an informational processing model that mimics the work of the neurons in the brain [5]. Their ability to learn quickly is what makes ANNs very powerful. This learning is done through an information flow that goes in two directions. Patterns from the training dataset are given to the ANN through the input neurons, then goes through the hidden layers and arrives to the output neurons.

Genetic Algorithms (GA) are considered the best solution for task and operation scheduling. They emerged from the research of Mr. John Holland conducted at the University of Michigan in 1960. However, it took them almost 30 years to become popular. The main purpose of GA is to solve complex problems where deterministic algorithms are considered an expensive solution. The Travelling Salesman Problem or the Knapsack problem are cases in point [6].

ANN and GA models are usually implemented in commodity computers or lately in Raspberry Pis. The NI CompactRIO is considered as a good alternative for deploying the ANN algorithms.

$\mathrm{NI}$ CompactRIO is a high-performance embedded controller with Input/Output modules. It has two targets: a real-time controller chassis, and an FPGA module. It includes a microprocessor to implement control algorithms and offer a support of a large pool of frequencies. The FPGA module is mainly used to accommodate for the high speed of certain modules and even certain programs. It deals with the data streaming from the I/O modules attached to the CompactRIO. The FPGA module is brought by Xilinx Virtex.

The CompactRIO is programmable using a specific graphical programming language named LabVIEW. This latter allows a better visualization of the data and an intuitive and easy way to implement control approaches.

In this paper, we are training an ANN model to predict the energy consumed by different appliances in a building. The model is developed in Python programming language but interfaced with LabVIEW for a potential integration in the NI CompactRIO.

The rest of the paper is organized as follows: Sect. 2 presents the scope of the research project under which this work is done. Section 3 contains the background of our work. In Sect. 4, we present the implementation steps and discussing the results obtained. Then, we conclude and present our future work in Sect. 5.

\section{Literature review}

A lot of work has been carried on in this area by different researchers in the community.

Authors in [7] are presenting a structure of a home energy management system to determine the best dayahead scheduling for the different appliances. This scheduling is based on the hour price and the peak power-limiting-based demand response strategies. In addition to that, they introduced a realistic test-case in order to validate their schedule. The test showed a significant drop in the energy consumed by the different appliances thanks to the schedule they designed.

Sou Kin Cheong et al. presented in [8] a scheduling method for smart home appliances based on mixed integer linear programming. Furthermore, they took into consideration the expected duration and peak power consumption of the appliances. Based on a previously defined tariff, the proposed schedule achieved about $47 \%$ of cost saving. Furthermore, the authors demonstrated that very good solutions can be obtained using very little computational power.

Regarding the energy consumption prediction, a good amount of work has been published representing different attempts to predict the energy consumed by different appliances. Elkonomou presented in [9] a prediction method based on artificial neural network. In order to select the best architecture, the multilayer perceptron model was used to make a set of tests to select the one with the best generalization. Actual data about input and output was used in the training, validation, and testing process.

Authors in [10] are stating the fact that the building energy consumption prediction is crucial for efficient energy planning and management. To do the prediction, they are presenting a model that is data-driven and that allows for the energy consumption prediction. The review shows that the area of energy consumption prediction has 
a good amount of gaps that require more research to be filled: the prediction of long-term energy consumption, the prediction of energy consumed within residential buildings, and the prediction of energy consumed by the lighting in buildings. The lack of research in these areas can be due to the relatively small amount of data that is available.

\section{Project scope}

The work done in this paper falls under the scope of a research project named MiGrid.

The project mainly aims at creating a holistic testbed platform that couples smart buildings and renewable energy storage and production. The general architecture of the project is depicted in the Fig. 1 [11].

The testbed has six main components:

1. Wireless sensors This refers to a set of wirelessly connected sensors forming a Wireless Sensor Network. This is responsible for sensing data in a specific context.

2. Wireless actuators These are wirelessly connected actuators that are supposed to translate electrical signals into mechanical actions to act on appliances.

3. Big data analytics platform This is a platform that takes care of the processing and the storage of data stemming from the entire SG.

4. NI CompactRIO It is considered as the main controller of the system. It decides on whether to inject the energy produced to the grid, store it, or use it to power loads.

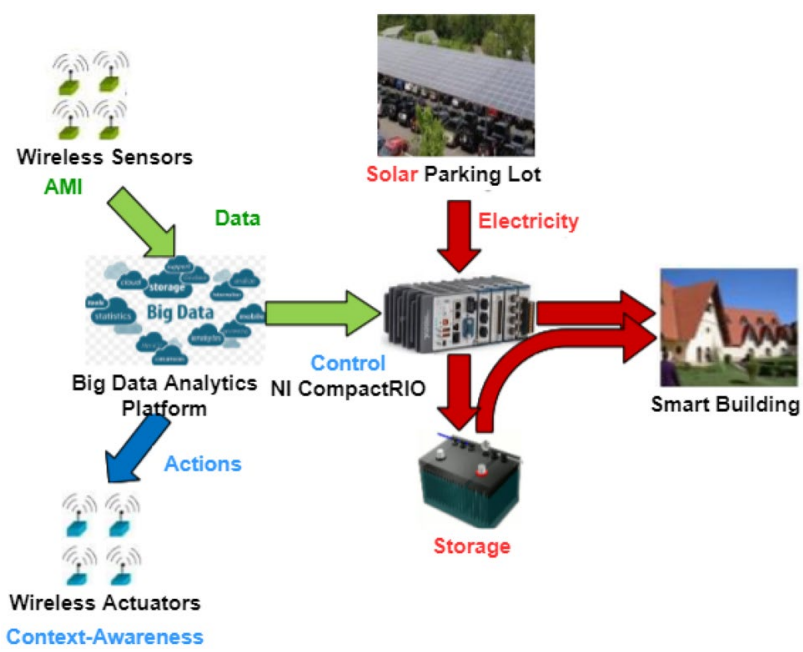

Fig. 1 MiGrid general architecture
5. Storage device This consists of a lithium battery that is supposed to store the excess of energy and make it available for use when needed.

6. Solar parking lot The main renewable energy source in the system.

\section{Background}

\subsection{Smart Grids}

By definition, a SG is nothing but an electrical grid that integrates a two-way communication system. In traditional electrical systems, electricity flows in one direction form the power plant to the home or business. The SG comes with an improvement to the network that resides in the on-the-spot feedback about the operations, power interruptions, and electricity consumption. This feedback is given back to the power plant and other operators [12].

The SG has the ability to tune itself to provide a better state of performance, and a better quality of the energy delivered. In addition to that, the SG is capable of anticipating problems and disturbances. Hence, the SG will allow for a more efficient transmission of electricity, lower kilowatt price, and quicker restoration.

The SG has three main components that are presented in Fig. 2.

- Physical power assets This consists of the power lines, transformers, etc. The information given by these assets is used by the smart meters and wireless devices, in addition to microgeneration and storage devices.

- Physical communication assets These are mainly the access and transport network (e.g. fiber) in addition to switches and routers. The main end users for this component are home area network.

- Software and applications This is mainly about the distributed data processing (on-site, off-site, and virtual-

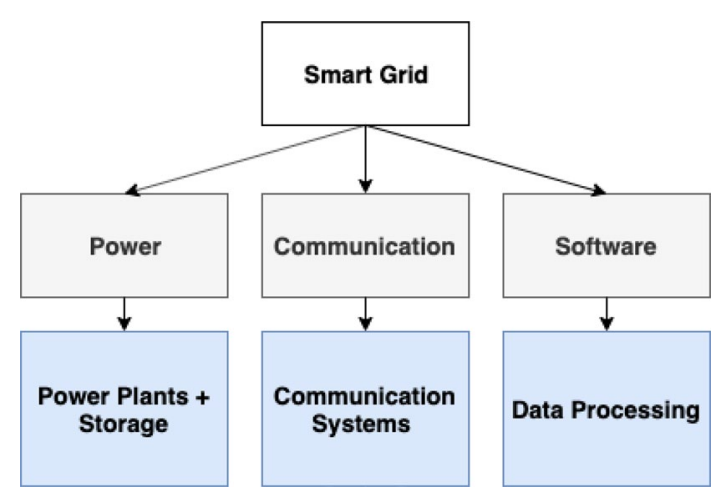

Fig. 2 Smart grid's components 
ized). This includes the management of the grid, the load balancing, IT security, and other roles.

\subsection{Energy management systems}

EMSs are supposed to reduce energy consumption up to $20-30 \%$. They are a set of connected hardware and software that have the following tasks:

- Monitoring Collection of energy consumption related information in order to establish the basis and clarify the changes in targets.

- Control Putting in place control algorithms in order to correct any deviations from the target.

The new EMS definition shows to be more peoplecentric than the older one. It engages users through connecting their actions with the energy consumption. The EMS gets the users involved by displaying their real-time energy consumption. Making the consumers aware of their daily energy usage can have a huge impact on their consuming behaviors [13].

EMSs are also well known for managing Microgrids whereby they have to process large amounts of data stemming from different sources. EMSs are supposed to use that data to control and monitor the Microgrid. Customers also can benefit from the EMS as it can provide them useful information inferred from the data generated by sensors (e.g. energy consumption, electricity prices, etc.) [14].

\subsection{Machine learning}

Machine learning is one of artificial intelligence (Al) applications. It involves providing systems with the ability to automatically learn and improve based on experiences without previous programming. This learning process is based on the exploration of large amounts of data.

It begins by closely observing the data to look for patterns that would help making better decisions. ML has a set of algorithms categorized as follows [15]:

- Supervised machine learning algorithms It applies what was learnt from past data to predict future events. The system provides, after sufficient training, targets for new inputs. This algorithm has the possibility to compare the predicted output to the actual one.

- Unsupervised machine learning algorithms It is used when the output needed to train the model is not classified or labeled. In other words, it infers a structure or pattern from hidden data.

- Semi-supervised machine learning algorithms These algorithms fall in between the previous two categories as they use both labeled and unlabeled data to train models.

\subsection{Time series forecasting methods}

Several methods can be used in the forecasting of time series data. Classical time series are somehow sophisticated and perform well on a wide range of problems. In this section, we will be exploring the different methods existing.

\subsubsection{Autoregression (AR)}

The Autoregression model (AR) is a model that predicts the behavior by learning from previous ones. It works well with data that is related to entries that precedes it and that follows it. To create the model, only the past data is used, hence the name autoregression. The process of creating the model is nothing but a linear regression between the current data and the past data.

The AR model is a stochastic model that has a certain degree of uncertainty and randomness associated to it. This means that the model developed and tested will never deliver a $100 \%$ accuracy. It gets only close enough to be used in prediction scenarios.

AR models are also referred to as conditional models, Markov models, or transitions.

$A R(p)$ models are autoregressive models where specific values are used to predict other values. The $p$ in $A R(p)$ is called the order, i.e. AR (1) is a regression model that is only interested in values that are one period apart. Eventually, models of second and third orders are related to values of two or three periods apart.

\subsubsection{Moving average}

A moving average (MA) is a well-known indicator that helps smoothing out actions by reducing the noise from random data. It is considered a trend-following because it is based on past data.

There are two main moving averages that are widely used: the simple moving average, and the exponential moving average. The simple moving average is the simple average of a security over a defined number of time slots. The exponential moving average focuses on the recent data entries and gives them higher weights. The most common applications of the MA are related to trend direction. This includes determining support and resistance levels.

Moving averages are useful enough by themselves, but they can also be combined with other technical indicators to deliver better results [16]. 


\subsubsection{Autoregression integrated moving average}

Autoregression integrated moving average (ARIMA) models are considered the most general class of time series forecasting models that can be used in conjunction with nonlinear alterations (e.g. logging).

There are two main models of ARIMA: seasonal, and non-seasonal models.

The main applications of this model are in areas of short-term forecasting which requires at least 40 past data points. It gives better results when the data used is stable over time with a minimum number of outliers.

\subsubsection{Artificial neural networks}

Artificial neural networks (ANN) are widely used in Machine Learning in general and in time series data forecasting in particular. ANNs are brain inspired and they intend to replicate the way that humans learn. They mainly consist of input and output layers in addition to hidden layers. ANNs are very powerful in finding patterns that are complex and numerous for humans to extract.

There are multiple types on ANNs, each one is used in specific scenarios and has a specific degree of complexity. The basic type is called feedforward neural network in which information travels in one direction (from input to output). Another widely used type is the recurrent neural network where data can flow in all directions. This type is used with complex tasks such as learning handwriting, face, and language recognition. Furthermore, convolutional neural networks and Boltzmann machine networks, and many others are used to solve machine learning problems.

ANNs learn in the same way that humans do: from past experiences. They require data to learn, and the more data they get, the more accurate the prediction. Data used to train ANNs is usually divided into three subsets: the training set which helps with establishing the required weights, the validation data set which helps fin-tuning the model, and the test set to see if the predicted output matches the actual output.

The main challenge that faces ANNs is the time needed to train the model, in addition to the required compute power that is needed for complex tasks. Furthermore, ANNs are considered black boxes that are fed with data to produce a certain output [17].

According to [18], neural networks perform better than many forecasting methods. Hence, we are opting for ANNs to develop our prediction model for energy consumption.

\subsection{Genetic algorithms for tasks scheduling}

A GA is a search method that is inspired by the theory of natural evolution. It mimics the natural selection process by selecting the fittest elements for the production of the next generation.

The natural selection process starts by identifying the fittest elements from the initial population. These elements produce what is called the offspring which inherits the characteristics of the parents and will be given to the next generation. If the fitness of the parents is good, the offspring will be better and hence the generations will survive. The same process keeps iterating until a new generation with the fittest elements is formed. This concept can be applied to search problems: a set of solutions is taken into consideration and the best solution is selected [19].

The GA goes through five phases:

- Initial Population: Each process starts with a set of elements (also referred to as individuals) called population. Each element of the population is an actual solution to the problem.

- Fitness Function It determines how fit each element is (i.e. its ability to compete with others). The function assigns a fitness score on which the probability of selection is based.

- Selection In this phase, the fittest elements are selected, and their genes are passed to the next generation.

- Crossover This is considered the most significant phase of the entire process. It consists of randomly choosing a crossover point from the genes of the parents to be mated.

- Mutation In the offspring formed, some random genes can be subject to a mutation. This means that some of the bits in the string can be flipped.

The algorithm keeps executing until the population converges (starts producing offspring that is similar to the previous ones). Then, we can say that the GA has given a set of solutions to the problem.

The GA cycle is represented in the loop in Fig. 3.

\section{Implementation and results}

\subsection{Data set}

To develop and train the model, we used a real-world dataset available online [20].

Some of the dataset's attributes are shown in the list below: 


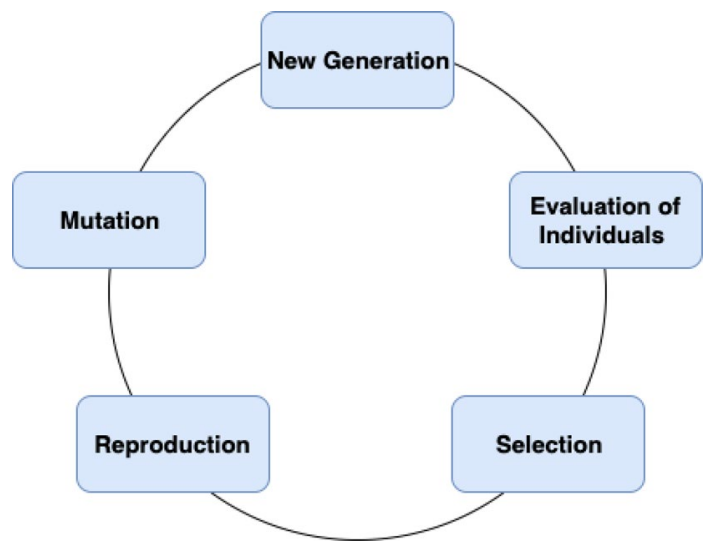

Fig. 3 Genetic algorithm cycle

Table 1 Data set sample

\begin{tabular}{lcccc}
\hline Timestamp & AC & Fridge & Furnace & Microwave \\
\hline 2018-07-30 9:00 & 1452.20 & 101.45 & 0 & 6.16 \\
2018-07-30 10:00 & 1153.47 & 133.70 & 149.11 & 37.95 \\
2018-07-30 11:00 & 1090.39 & 38.67 & 145.18 & 6.15 \\
2018-07-30 12:00 & 655.75 & 57.76 & 87.99 & 38.93 \\
2018-07-30 13:00 & 1626.13 & 63.43 & 190.76 & 6.20 \\
2018-07-30 14:00 & 2138.96 & 0 & 233.55 & 5.45 \\
2018-07-30 15:00 & 1635.82 & 94.14 & 186.96 & 10.43 \\
2018-07-30 16:00 & 2145.36 & 33.52 & 233.68 & 6.86 \\
\hline
\end{tabular}

- Timestamp it shows the date and time when the energy consumption was recorded.

- Main_ $i$ the energy consumption of the main room.

- Ac the energy consumption of the air conditioner.

- Living_rm the energy consumed by the living room appliances.

- Fridge the energy consumption of the fridge.

- Microwave the energy consumed by the microwave.

- Furnace energy consumed by the heaters.

For our model, we used only data about the AC, fridge, furnace, and microwave. A sample of the data used is shown in Table 1.

\subsection{Artificial neural network model}

To build the prediction model, we opted for a feedforward artificial neural network with two input neurons, ten hidden neurons and one output neuron.

The ANN we are using is depicted in Fig. 4.

The model designed has as input the time stamp when the energy consumption has been recorded, and the appliance. For our case, we will be assigning a unique ID

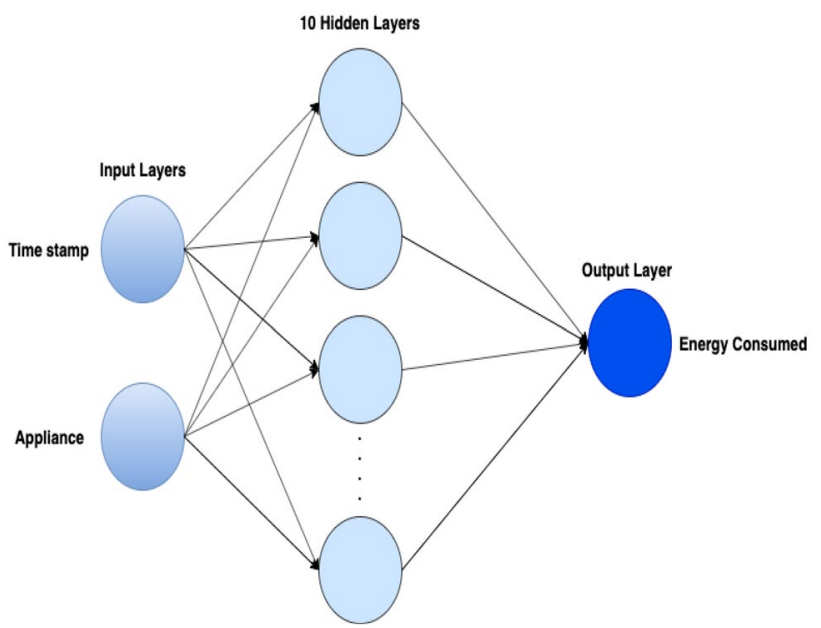

Fig. 4 ANN model

Table 2 Appliances'IDs

\begin{tabular}{ll}
\hline ID & Appliance \\
\hline 1 & AC \\
2 & Fridge \\
3 & Furnace \\
4 & Microwave \\
\hline
\end{tabular}

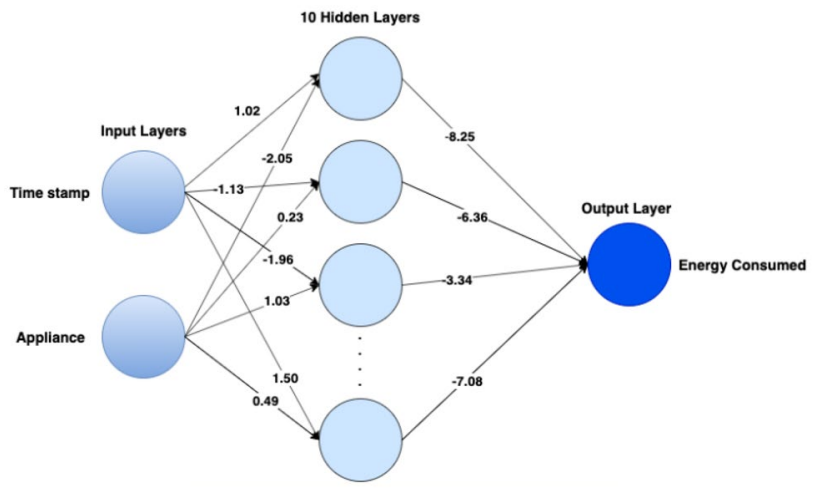

Fig. 5 ANN after first iteration

to each appliance and then feed it to the neural network. The appliances' IDs are shown in the next table (Table 2).

The output layer of the model designed consists only of the energy consumed by each appliance.

Our input data is a $10 \times 2$ matrix ( 10 being the number of hidden neurons, and 2 being the number if input neurons), and the output data is a $10 \times 1$ matrix ( 1 being the number of output neurons).

After the first iteration, our model looks like Fig. 5.

To calculate the error of our model, we represented the loss function by using the mean sum squared loss. The 


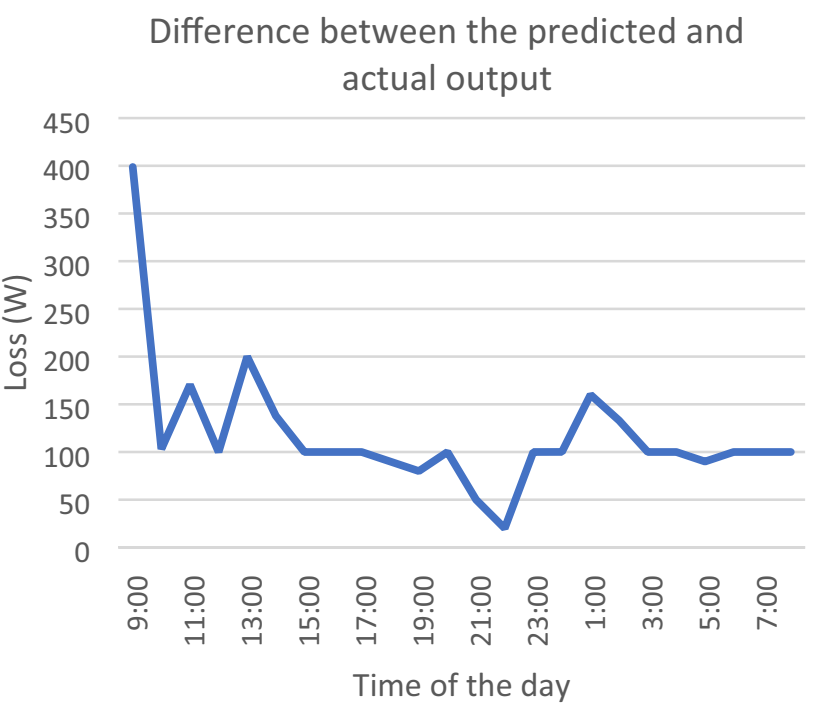

Fig. 6 ANN model loss

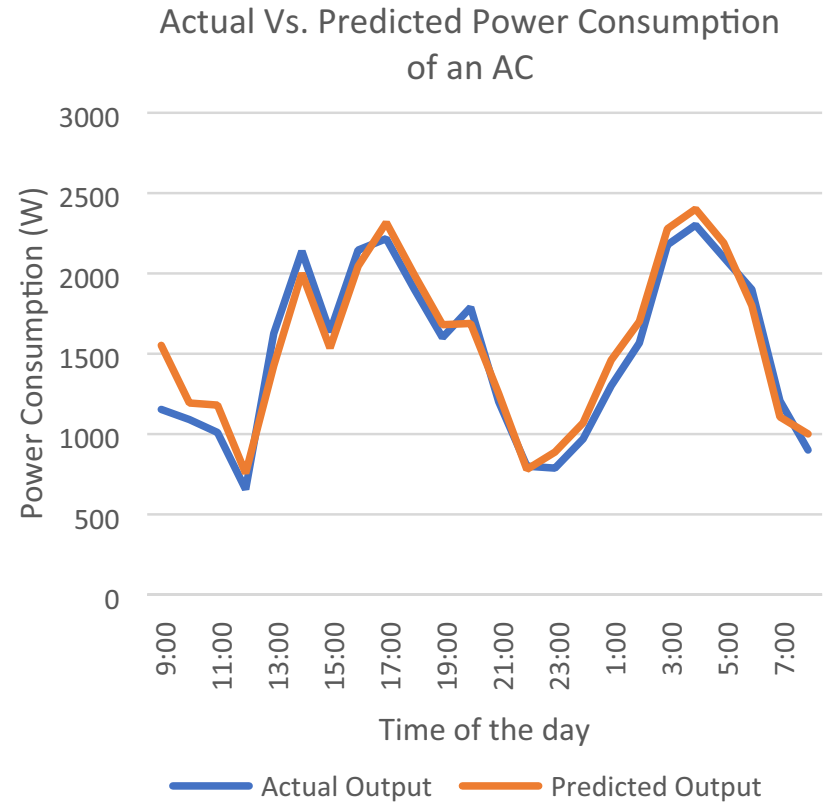

Fig. 7 Actual versus predicted output

loss value obtained through the training iterations is represented in Fig. 6.

The loss is found to be between $2.5 \%$ and $36 \%$ of the actual output.

The actual and predicted outputs are plotted in the Fig. 7.

The study in [21] used artificial neural networks for the prediction of Greek long-term energy consumption. For this purpose, the authors made use of the multilayer perceptron model was investigated in order to test the

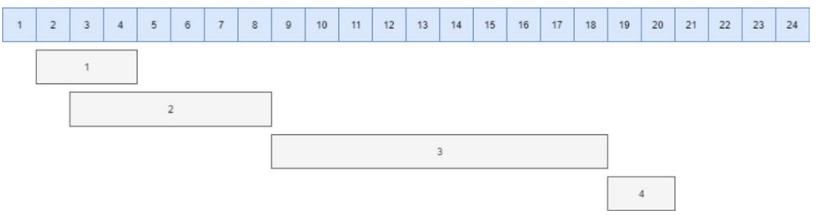

Fig. 8 Appliances'schedule

Table 3 Schedule of different appliances

\begin{tabular}{llcc}
\hline ID & Appliance & Start time & End time \\
\hline 1 & AC & 2 & 5 \\
2 & Fridge & 3 & 9 \\
3 & Furnace & 9 & 19 \\
4 & Microwave & 19 & 20 \\
\hline
\end{tabular}

different available architectures, and eventually select the optimal one. Furthermore, other prediction methods have been used, namely the linear regression and the support vector machine model. These models were compared to the ANN in terms of the accuracy.

The ANN model described showed a highest accuracy among all the tested prediction methods. The percentage error of the model was about $2 \%$. This is mainly due to the high quality of the data used to train and validate the model. While we used data from an online source, the authors of this work have collected real records which had a great impact on the model output.

\subsubsection{Genetic algorithm model}

The GA model we are using takes into consideration the four appliances mentioned previously in this paper (i.e. AC, Fridge, Furnace, and Microwave).

Each appliance has a specific start time, working duration, and eventually a specific end time. These parameters are described in the diagram below (Fig. 8).

According to the diagram above, we have four resources that need to be scheduled daily. By scheduled, we mean for each appliances or resource, the start and end time need to be determined for each day (Table 3).

The data fed to the GA is represented as follows:

The GA model used is scored-based. Each combination is assigned a score which determines how accurate the schedule is. Hence the best schedule should have a score equal or closer to 0 .

After training the model, we have the following schedule.

- Score-5.

- Start_times $[0,4,8,12,16]$. 
- Appliances $[1,2,2,3,4]$.

\subsubsection{Deployment in CompactRIO using LabVIEW}

Programming CompactRIO with LabVIEW is done through what is called a Virtual Instrument (VI). It has two components: a front panel and a block diagram. The front panel contains the design part of the VI. This means it includes buttons, labels, and indicators. The block diagram shows the actual wiring of the different components in the instrument.

Since we are integrating python with LabVIEW, we are following the architecture in Fig. 9. The VI is to be deployed later on the CompactRIO controller.

In the architecture presented above, the user is supposed to provide the program with input (mainly the time and the appliance ID for the energy consumption prediction). As a standalone python application, the input is given through the system arguments. After the integration with the LabVIEW VI, the input is given to the application through numeric controls implemented in the VI.

The inputs are then used by the python scripts through a LabVIEW library called "exec".

Once the scripts finish execution, the results are shown in a numeric indicator.

The front panel of the VI used for this purpose is shown in Fig. 10.

In the front panel of the VI, we have two numerical controls where the user enters the time and the ID of the appliance. In addition to that, we introduced a numerical indicator that displays the predicted energy consumed at the time entered for a specific appliance.

The block diagram of the VI is shown in Fig. 11.

The VI has four main components. We are describing them below:

1. Build path to Python script, which is expected to be in the same folder as this VI. The Python script could be moved, but this VI would have to reflect that change.

2. Create command line argument, in the form of pythonpath scriptpath int32 int32. Quotation marks

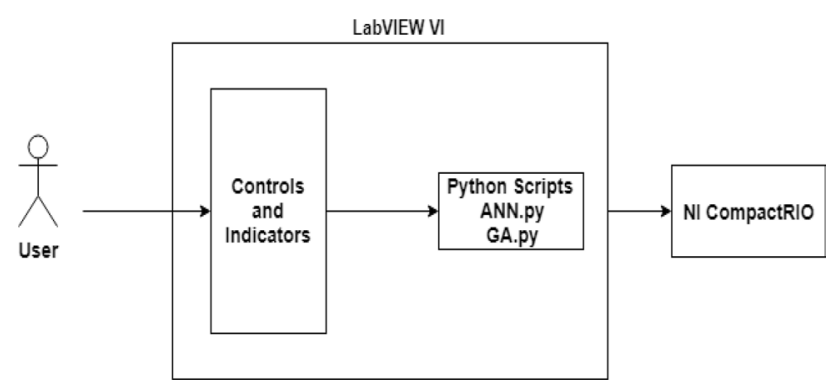

Fig. 9 Program architecture are used for the path, in case there are spaces in the path.

3. Execute the argument. The run in minimized, so that the command window does not quickly appear and disappear.

4. Convert the string output from Python to an int.

\section{Discussion}

As you may have noticed from the results, the models did not show a very high accuracy compared to other existing models. The loss of our ANN model is relatively high which makes it a non-reliable method for the energy consumption prediction. This is mainly due to the quality of data used. Our data did not go through any preprocessing.

Also, as ANNs need to learn from the data provided, our dataset did not help the ANN to learn and hence the final model was poor.

There is a huge ongoing debate that is taking place between practitioners on where to go for ANNs or statistical methods for the prediction. The article in [22] contains a study that compares ANNs to regression with a medium to large dataset of patients (size $>200$ ). According to the review, ANN outperformed the regression method in $36 \%$ of the cases and was outperformed in $14 \%$ of them.

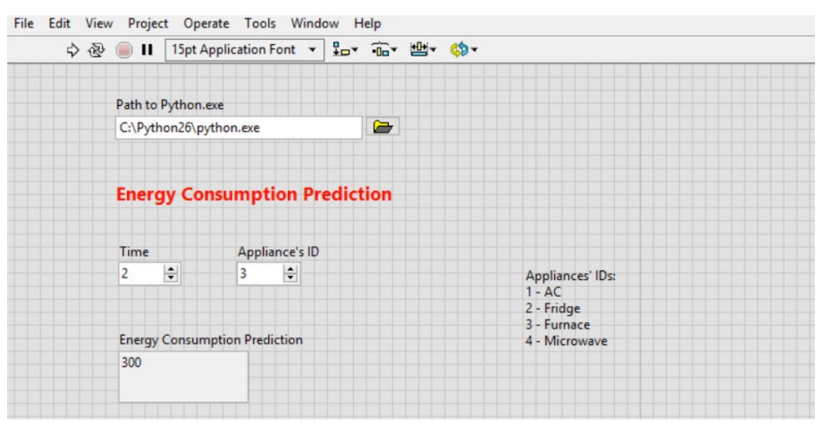

Fig. 10 Front panel of the VI

File Edit View Project Operate Tools Window Help

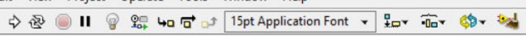

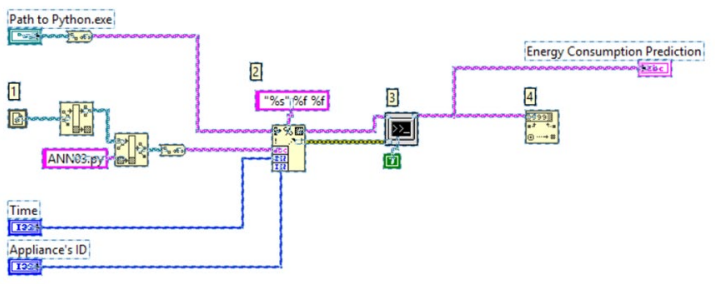

Fig. 11 Block diagram of the VI 
However, as the dataset size increased (size $>5000$ ), regression showed a better performance.

Authors in [23] have conducted a comparative study between ANNs and statistical methods in the prediction of consumer's behavior. As results, they found that ANNs constantly win the battle against other methods. Due to the flexibility of the models, they were able to perform better with both known and unknown choice rules. The ANN model used in this study is iterative, this implies that the model learns complex attributes about consumers.

ANNs are different from other statistical methods in the was that they do not expect any relationship between the input and output variables. However, the model overfitting can represent a real problem when conceiving an ANN model. Hence, one needs to make sure that the data fed to the model is preprocessed.

\section{Conclusion and future work}

In this paper, we delineated the details of deploying a machine-learning-based model for energy consumption prediction and scheduling in Smart Buildings. The presented model can serve as a roadmap for deploying real-world SB testbeds, and thus paving the way towards grounded research around Smart Buildings for Smart Grids.

We integrated the models using python in a LabVIEW program by creating a VI instance that allows the user to select an appliance, along with the time of the day, and then compute relevant predicted consumption using our ANN-based model. The models did not show very high accuracy as the dataset used was not that big enough for training and validation. When looking, and analyzing, similar models in the literature using ANNs for prediction, we came to the conclusion that ANNs are not always the best and they can be outperformed by basic statistical methods, regression being a case in point. Nevertheless, this remains an ideal venue to investigate for researchers seeking machine learning as long as they can afford good data, ideally a big one.

As a future work, we intend to collect further data from our testbed, and get some relevant big data as well, in order to tailor better models. In our testbed, we are intending to further gather PV production data, energy consumption of different appliances, and the context data (i.e. motion, temperature, humidity, etc.). Last, but not least, we will deepen our investigation of existing prediction methods by namely incorporating statistical approaches as well.

Acknowledgment This work is sponsored by US-NAS/USAID under the PEER Cycle 5 project grant\# 5-398, entitled'Towards Smart Microgrid: Renewable Energy Integration into Smart Buildings".

\section{Compliance with ethical standards}

Conflict of interest The authors declare that they have no conflict of interest.

\section{References}

1. SmartGrids.gov, What is the Smart Grid? [En ligne]. https://www. smartgrid.gov/the_smart_grid/smart_grid.html. [Accès le 22 April 2019]

2. Arcos-Aviles Diego et al (2016) Fuzzy logic-based energy management system design for residential grid-connected microgrids. IEEE Trans Smart Grids 9(12):530-543

3. Mocanu Elena et al (2016) Unsupervised energy prediction in a Smart Grid context using reinforcement cross-building transfer learning. Energy Build 116:646-655

4. Jordan I et al (2015) Machine learnig: trends, perspective, and prospects. Science 349(16245):255-260

5. Dacombe J (2017) An introduction to artificial neural networks (with example), 23 Oct 2017. [Online]. https://medium.com/@ jamesdacombe/an-introduction-to-artificial-neural-networkswith-example-ad459bb6941b. Accessed 22 Apr 2019

6. Sablier (2019) Genetic algorithms [Online]. https://www.codin game.com/playgrounds/334/genetic-algorithms/history. Accessed 1 May 2019

7. Paterakis NG, Erdinç O, Bakirtzis AG, Catalão JPS (2015) Optimal household appliances scheduling under day-ahead pricing and load-shaping demand response strategies. IEEE Trans Ind Inform 11(16):1509-1519

8. Cheong SK et al (2011) Scheduling smart home appliances using mixed integer linear programming. In: 50th IEEE conference on decision and control and European control conference, IEEE. https://doi.org/10.1109/cdc.2011.6161081

9. Ekonomou L (2010) Green long-term energy consumption prediction using artificial neural network. Energy 35(12):512-517

10. Amasyali $\mathrm{K}$ et al (2018) A review of data-driven building energy consumption prediction studies. Renew Sustain Energy Rev 81(11):1192-1205

11. Abid MR, Lghoul R, Benhaddou D (2017) ICT for renewable energy integration into smart buildings: IoT and big data approach. In: IEEE Africon, pp 883-888. https://doi.org/10.1109/ afrcon.2017.8095594

12. Marc Lanllanilla SW (2013) What is a smart grid? LiveScience, 12 Dec 2013 [Online]. https://www.livescience.com/41920-smart -grid.html. Accessed 23 Apr 2019

13. Atin (2013) Energy managment system, 17 Sept 2013 [Online]. http://www.sensegrow.com/article/what-energy-management -system. Accessed 25 Nov 2019

14. Talei H, Essaidi M, Benhaddou D (2018) An end to end real time architecture for analyzing and clustering time series data: case of an energy management system. In: 6th international renewable and sustainable energy conference (IRSEC), 2018

15. Expert System (2017) What is machine learning? A definition, 3 Mar 2017 [Online]. https://expertsystem.com/machine-learn ing-definition/. Accessed 3 Dec 2019

16. Hayes A (2019) Moving average (MA), 04 Apr 2019 [Online]. https://www.investopedia.com/terms/m/movingaverage.asp. Accessed 27 Apr 2019

17. Dormehl L (2019) What is an artificial neural network? Here's everything you need to know. Digital Trends, 05 Jan 2019 [Online]. https://www.digitaltrends.com/cool-tech/what-is-anartificial-neural-network/. Accessed 27 Apr 2019 
18. Hill T et al (1996) Neural network models for time series forecasts. Manag Sci 42(17):1082-1092

19. Mallawaarachchi V (2017) Introduction to genetic algorithmsincluding example code, 08 July 2017 [Online]. https://towar dsdatascience.com/introduction-to-genetic-algorithms-inclu ding-example-code-e396e98d8bf3. Accessed 01 May 2019

20. Ewood (2016) Home energy consumption, 05 Aug 2016 [Online]. https://data.world/ewood/home-energy-consumption/works pace/file?filename=download+\%288\%29.csv\#. Accessed $27 \mathrm{Apr}$ 2019

21. Ekonomou L (2009) Greek long-term energy consumption prediction using artificial neural network. Energy 35(1):512-517
22. Sargent DJ (2001) Comparison of artificial neural networks with other statistical approaches. Cancer 91(S8):1636-1642

23. West Patricia M et al (1997) A comparative analysis of neural networks and statistical methods for predicting consumer choice. Mark Sci 16(14):370-391

Publisher's Note Springer Nature remains neutral with regard to jurisdictional claims in published maps and institutional affiliations. 